\title{
MORPHOLOGICAL DESCRIPTIONS USING THREE-DIMENSIONAL WAVEFRONTS
}

\author{
JEAN SERRA \\ Centre de Morphologie Mathématique, Ecole des Mines de Paris, 35, rue Saint Honoré, 77300 Fontainebleau, \\ France \\ e-mail: serra@cmm.ensmp.fr \\ (Accepted June 18, 2002)
}

\begin{abstract}
The present study deals with the analysis of three-dimensional binary objects whose structure is not obvious nor generally clearly visible. Our approach is illustrated through three examples taken from biological microscopy. In one of our examples, we need to extract the osteocytes contained in sixty confocal sections. The cells are not numerous, but are characterized by long branches, hence they will be separated using a directional wavefront. The two other objects are more complex and will be analysed by means of a spherical wavefront. In the first case, a kidney of a rat embryo, the tissue grows like a tree, where we want to detect the branches, their extremities, and their spatial arrangement. The wavefront method enables us to define precisely branches and extremities, and gives flexible algorithms. The last example deals with the embryonic growth of the chicken shinbone. The central part of the bone (or shaft) is structured as a series of nested cylinders following the same axis, and connected by more or less long bridges. Using wavefronts, we show that it is possible to separate the cylinders, and to extract and count the bridges that connect them.
\end{abstract}

Keywords: 3-D geodesy, branching, Euler-Poincaré constant, wavefronts.

\section{EXPERIMENTAL CONTEXT: THREE-DIMENSIONAL MICROSCOPY}

The approach we present here was born from practical cases, more precisely from two separate issues in three-dimensional optical microscopy which were presented to me, independently, after a space of one month.

\section{FIRST ISSUE: THE KIDNEY}

In February 2000, Dr. John Bertram, ${ }^{1}$ nephrologist and serving Chairman of the International Society for Stereology, spent two days at the CMM, during which he presented his current work. The subject of his research is the embryonic development of the kidney studied in animals such as the rat (Clark et al., 2001). He takes advantage of the property of embryonic kidney to develop in vitro, which enables him to study the organ evolution by confocal microscopy without animal destruction (Fricout et al., 2002).

Dr. Bertram left us the serial sections of two kidneys, and proposed to work with a student from the Ecole des Mines, for developing a morphological approach to his problem. Hence the decision to launch an internship for Gabriel Fricout.
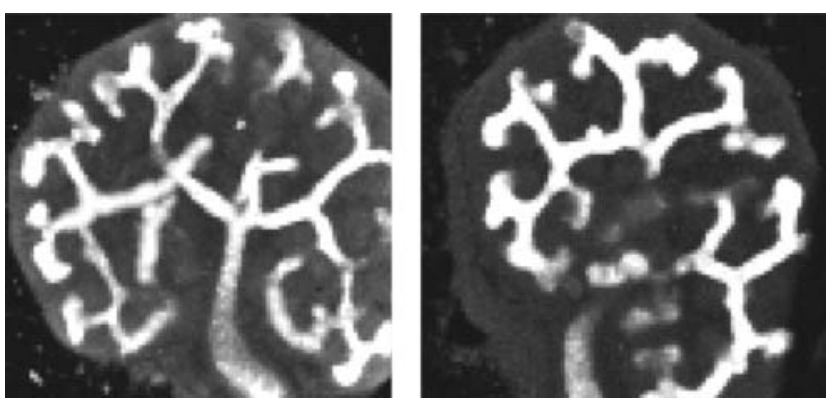

Fig. 1. a) Kidney under study (micrograph of confocal microscopy); b) other kidney specimen. The value at each pixel represents the maximum grey tone, for this pixel, over the 29 sections. How to extract the branches and the extremities of such a tree?

We can see in Fig. 1 an image of each kidney before binarization, showing that the structure develops in the form of a tree. The expected morphological description bears on the geometry of the tree, and involves two objects:

- extremities: where are they located? how are they arranged in space?

- branches: where are they located? according to which hierarchy and length?

Confocal microscopy reveals here a highly anisotropic sample. Each series contains 29 sections

\footnotetext{
${ }^{1}$ Dpt of Anatomy, Faculty of Medicine, Univ. of Melbourne, Parleville, Victoria 3052, Australia
} 
$30 \mu \mathrm{m}$ thick, in which the orientation is roughly perpendicular to the trunk.

On each section, the pixels are arranged according to a square grid, whose spacing is about $4 \mu \mathrm{m}$. The digital volume element (voxel) looks like a cylinder with a square base, which is seven times as high as it is wide. Each branch extremity is surrounded by nephrons (i.e. the basic filtering structure of the kidney), whose number is indicative of the future capacity of the fully-grown kidney. The nephrons, which cannot be seen here, will become visible through a future double staining. Then, we will have to study the relationship between the shape of the tree and the number of nephrons it can receive.

The discussion that followed Dr Bertram's talk showed that F. Meyer, S. Beucher and I did agree on avoiding 3-D skeletons, and on approaching the problem by means of 3-D geodesy. Indeed, 3-D skeletons are not made of lines, as in the 2-D case, but combine lines with flat portions; in addition, they are more sensitive to barbs and to irregularities than in two dimensions. Nevertheless, the student adopted a skeleton-based method, but in 2-D, and associated with a 3-D back projection (Fricout et al., 2002), i.e. a tailormade approach for the images under study. However, one month later, when the shinbone problem arose, it became obvious that 3-D geodesy only could provide a general framework.

\section{SECOND ISSUE: THE SHINBONE}

Dr. Staub studies the morphogenesis of long bones, and works on the shinbones of chicken embryos. He designed a dynamic model of the long central zone (shaft), where the compact future bone appears as a series of nested co-axial cylinders ${ }^{2}$ (see Fig. 2). For verifying the model, an experiment conducted by $\mathrm{M}$. Mendjeli has consisted in slicing the shinbone shaft, perpendicularly to its axis, into a series of a hundred semi-thin sections, roughly like slicing a sausage. It results into a nearly cubic grid of voxels whose step is close to $1 \mu \mathrm{m}$ and whose size is approximately $300 \times 300 \times 100$.

Unlike the previous example, the primary difficulty here is to detect the object under study. The nested cylinders are not directly visible, and one has no idea of the number of tunnels and cavities they may contain. However, the bone image is virtually binary. Finally, as in the preceding case, the space is "oriented" from a marker, and the central marrow space plays in this second example the same role as the contact zone between the kidney and the gelatine (bottom of the tree) did in the in the first example. Is it possible to segment the concentric cylinders of the bone, and to describe them in quantitative terms (thickness, porosity, contacts between cylinders, etc ...)?
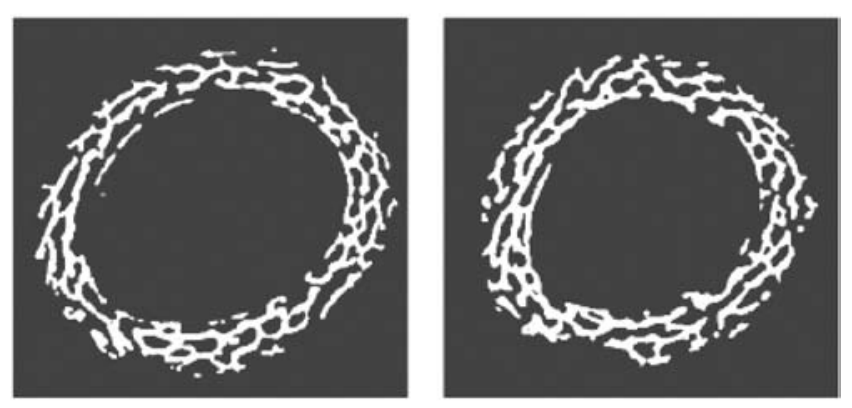

Fig. 2. Two horizontal sections of a shinbone epiphysis (chiken embryo). They come from a series of one hundred semi-thin sections. Is the shinbone made of nested cylinders?

\section{METHOD: WAVEFRONTS}

\section{CHOQUET'S THEOREM}

When a stone is thrown into a lake and generates a disturbance, a wave string is being created and spreads out while going around the possible obstacles, until the most remote points from the middle. The wavefront, circular in the case of a lack of borders, laps the islands and the lake contours and finally covers completely the lake, as shown in Fig. 3. More formally, the wavefront $F(\lambda, x)$ is defined as the zone of the space reached at time $\lambda$ when the wave stemming from point $x$ has a unit speed (Serra, 1988).
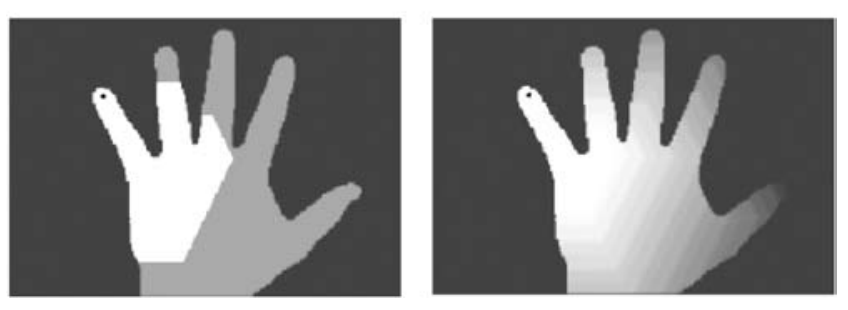

Fig. 3. 2-D geodesics. Point $x$ is the black point in the small finger, and the reference mask $Z$ is the hand. a) Geodesic disc $B(\lambda, x)$ centered at point $x ; b)$ Geodesic distance function from $x$.

In order to extract connected objects selected by markers, Meyer (Meyer, 1976) and Klein (Klein, 1976) were the first ones to transfer these notions to mathematical morphology, and the very first formalization, named "geodesic metrics" was established by Lantuejoul and Beucher (Lantuejoul et

\footnotetext{
${ }^{2}$ Verbal communication of Dr Staub, Laboratoire de recherches orthopédiques, CHU Laroboisière-St Louis, 75010 Paris, France.
} 
al., 1981). Indeed, in Fig. 3, the zone of the reference set, or mask, $Z$, swept between instants 0 et $\lambda$ by the wavefront born from point $x$ at the original instant turns out to be a disk $B(\lambda, x)$ of radius $\lambda$ for the geodesic metrics. This disk is smaller than the Euclidean one of the same radius and completely contained in $Z$. When the reference set $Z$ is compact, the induced metrics $\{B(\lambda, x), x \in Z\}$ satisfy the following property, derived from Choquet's Theorem 11-6 in Choquet (1966)

Theorem 1 Let $Z$ be an Euclidean compact set and let $x$ and $y$ be two disjoint points of $Z$. If there exist curves of finite lenghts with extremities at points $x$ and $y$ respectively, and if $\lambda$ stands for the lower limit of their lengths, then there exists an arc included in $Z$ whose length is $\lambda$ and whose extremities are $x$ and $y$, respectively.

Remember that in the Euclidean space, a set is compact when it contains its boundary and when one can include it in a square of finite side. In what follows, we will always suppose that reference sets $Z$ are connected and compact, and that for any points $x, y$ selected in $Z$, there is an arc with a length bounded by a $\lambda_{\max }(Z, x)$ and linking these two points. This precaution is meant to exclude compact sets such as, for instance, a spiral which winds indefinitely around a circle.

\section{THE ULTIMATE ELEMENTS OF THE WAVEFRONTS}

This section takes up C. Lantuejoul's and S. Beucher's results (Lantuejoul et al., 1981), but presents them differently. When using geodesics, it becomes possible to associate any point $x \in Z, Z \in \mathbb{R}^{n}$, with the point or points $y \in Z$ which are the furthest away from $x$. Indeed, let $\stackrel{\circ}{B}(\lambda, x)$ be the geodesic open ball of radius $\lambda$ and centre $x$, and $\lambda_{0}$ be the upper limit of the $\lambda$ such that $\stackrel{\circ}{B}(\lambda, x)$ be strictly contained in $\mathrm{Z}$. As the non empty compact sets $\left\{Z \backslash \stackrel{\circ}{B}(\lambda, x), \lambda<\lambda_{0}\right\}$ decrease and that $\mathbb{R}^{n}$ is a separated space, the intersection

$$
\underset{\lambda<\lambda_{0}}{\Gamma}[Z \backslash \stackrel{\circ}{B}(\lambda, x)]
$$

is itself a non empty compact set, whose points are all at the maximum distance $\lambda_{0}$ from $x$. This intersection is named "geodesic ultimate eroded set", and $\stackrel{\circ}{B}\left(\lambda_{0}, x\right)$ is the "geodesic ultimate dilated set" of point $x$.

The existence of extreme points may also be considered in a regional framework, and not a global one anymore. We must suppose that, $Z$ and $x$ being given, it is possible to find a $\mu(Z, x) \leq \lambda_{0}(Z, x)$ such that each connected component of $Z \backslash \stackrel{\circ}{B}(\lambda, x), \mu \leqslant$ $\lambda \leqslant \lambda_{0}$ decreases without subdividing. Then, the previous analysis should simply be applied to sets

$$
K_{i} \cap[Z \backslash \stackrel{\circ}{B}(\lambda, x)] \quad \mu \leqslant \lambda \leqslant \lambda_{0}
$$

where the $K_{i}, i \in I$ refers to the connected components of $Z \backslash \stackrel{\circ}{B}(\mu, x)$. Therefore, we obtain the farthest connected components from point $x$, such as, for instance, the fingers tips for $x$ taken around the middle of the wrist.

Both algorithm families about geodesics correspond to both our points of view. Invasion by geodesic balls led to all the particles reconstruction variants (deletion of the grains crossing the field border, hole filling, individual analysis, etc ...) and the search for extreme residues led to the ultimate eroded points, to the objects limits and to the length of a connected component (as a supremum of the distances between pairs of extreme points).

\section{DIGITIZATION}

The digitization of geodesic operations may cause errors, but limited ones; indeed, it is advisable to choose, as a circle or unit sphere, the closest shapes to their Euclidean homologues. Therefore, in 2D the hexagon, whose six vertices are equidistant from the center is better than the square, and, for the same reason, the cube-octahedron is better than the cube in 3D Meyer (1992).

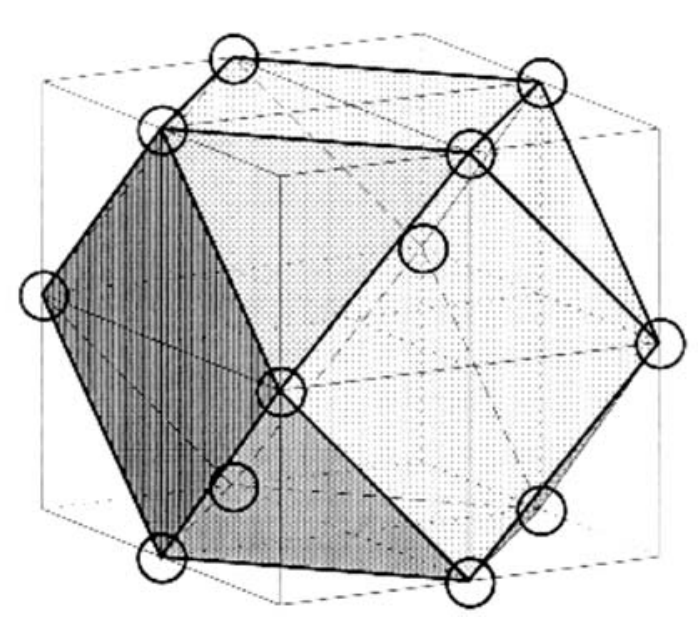

Fig. 4. Cube-octahedron.

This $\mathbb{Z}^{3}$ ball is very easy to build, when a numerical data network in square grid is available (Serra, 1997). 
It suffices to shift all even planes by half a diagonal of the unit cube (any diagonal, but always the same one). In practice, data are of course not moved, but only structuring elements. For example, the substitute for the 13 voxels of the regular cube-octahedron (Fig. 4) is calculated by dilating the central point according to the staggered unit cube-octahedron presented in Fig. 5. It requires three successive planes and differs whether the center lies in an even plane or in an odd one.

a)

upper and lower planes

$$
\begin{aligned}
& \text { central plane } \\
& \left(\begin{array}{ccc}
\cdot & \mathbf{1} & \cdot \\
\mathbf{1} & \mathbf{1} & \mathbf{1} \\
\cdot & \mathbf{1} & \cdot \\
\cdot & \mathbf{1} & \cdot \\
\mathbf{1} & \mathbf{1} & \mathbf{1} \\
\cdot & \mathbf{1} & \cdot
\end{array}\right)
\end{aligned}
$$

Fig. 5. Staggered successive planes, for simulating a unit cube-octahedron on a cubic grid. When the serial number of the central plane is odd (resp. even), the corresponding unit cube-octahedron is provided by configuration a) (resp. b).

The wavefront emanating from this central point starts with the point's 12 neighbours; when the interplane equals $a / \sqrt{2}(a=$ square grid spacing of the horizontal planes), the structure becomes completely isotropic and the 12 neighbours are equidistant from the center. This will be our assumption (section 5) about the shinbone, but this hypothesis is not essential, and, in any case, cannot be ventured for the study about embryonic kidneys (section 3).

The switch from the unit ball $C(x)$ of $\mathbb{Z}^{3}$ (octahedron, prism or cube) to its geodesic version $B_{1}(x)$ inside a mask $\mathrm{Z}$ is

$$
B_{1}(x)=C(x) \Gamma Z
$$

and the geodesic ball $B_{x}(x)$ of the size is obtained by $\mathrm{n}$ iterations of the previous one:

$$
B_{n}(x)=B_{1}\left[B_{n-1}(x)\right]\ulcorner Z
$$
equals

The corresponding wavefront, or geodesic sphere

$$
F_{n}(x)=B_{n+1}(x) \backslash B_{n}(x)
$$

\section{WAVEFRONTS AND TREE DIAGRAMS}

Let $Z$ be a compact set in $\mathbb{R}^{n}$ and $x \in Z$ be a point in $Z$. We propose to study the evolution of the connected components number of the wavefront $F(\lambda, x)$ when, as $\lambda$ increases, the compact space $\mathrm{Z}$ is swept. The two types of branching, division or confluence, supposedly remain in finite number when $\lambda \in\left[0, \lambda_{\max }\right]$, so that for any branching at $\lambda=\lambda_{0}<\lambda_{\max }$; it is always possible to find an open interval $] \lambda_{1}, \lambda_{2}\left[\right.$ containing $\lambda_{0}$, and inside which there are no other branching. The number of branches which may gather in $\lambda_{0}$ is supposed to be finite. Finally, as the branching may take the two dual shapes (division or confluence) when $\lambda$ increases, it is conventionally agreed in the proof below that the passage $\lambda_{1} \rightarrow \lambda_{2}$ corresponds to a division.

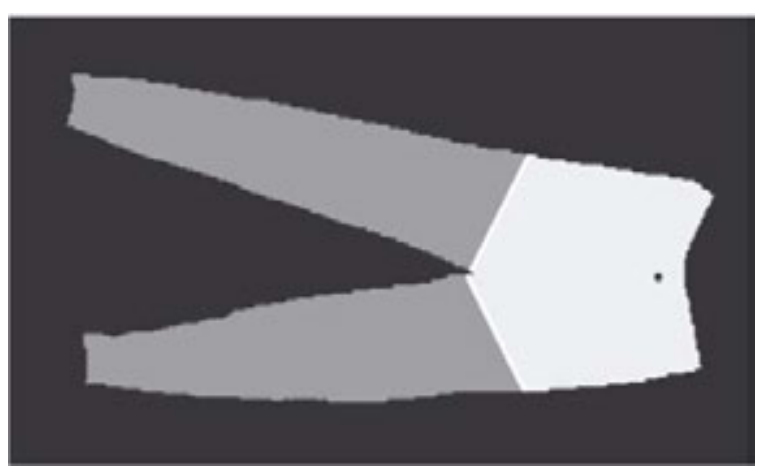

Fig. 6. Example of branching.

Therefore, we are led to the situation described in Fig. 6 , where point $\mathrm{x}$ is in black, the open ball $\stackrel{\circ}{B}\left(\lambda_{0}, x\right)$ in light grey, its complement $K\left(\lambda_{0}\right)$ in $Z$ in dark grey, and where the white wavefront indicates the precise moment of the branching. So, the compact set

$$
K(\lambda)=Z \backslash \stackrel{\circ}{B}(\lambda, x)
$$

has a unique connected component, when $\lambda<\lambda_{0}$; and more when $\lambda>\lambda_{0}$. In order to determine what happens when $\lambda=\lambda_{0}$, we first observe that for compact sets, we have $\Gamma\left\{K(\lambda), \lambda<\lambda_{0}\right\}=K\left(\lambda_{0}\right)$.

The compact $K\left(\lambda_{0}\right)$ is composed of only one connected component. Otherwise, they would be separated by a minimum distance $d$; but this is incompatible with the fact that, for any dilation of size $\varepsilon$, with $0<\varepsilon<d$, the geodesic dilate of $K\left(\lambda_{0}\right)$ becomes connected. Therefore, the front $F\left(\lambda_{0}, x\right)$ itself is connected, as otherwise, to switch from one of its components to another one, it would be necessary to cross a $K(\lambda)$ with $\lambda>\lambda_{0}$, but these $K(\lambda)$ are not connected anymore.

When $\mathrm{Z}$ has several branchings, the same description applies for each branch, upwards or downwards from the propagation from point $x$, which consequently partitions the set $\mathrm{Z}$ into a series of successive pieces.

The case of the $\mathrm{X}$ branching has also to be considered. It occurs when at least two branches stop 
at the critical front, and at least two of them start from there. In this case, the intermediary connected region is reduced to the front in $\lambda_{0}$, for, if it was larger, we would come back to the previous case; and if the front was not taken into accout, we would no longer have a critical element, but only separated branches. By gathering these results, we can state:

Proposition 2 Let $Z$ be a compact of $\mathbb{R}^{n}$. If, for any point $x \in Z$, the wavefront $F(\lambda, x)$ emanating from $x$ admits a finite number of connected components, with a finite variation, then, as radius $\lambda$ varies, $F(\lambda, x)$ partitions $Z$ into a finite number of connected sections, corresponding to open intervals of $\lambda$, and separated by connected components of the front which are located at the critical points of the branchings.

It is easy to prove that this partition has the largest possible that satisfies the properties of the proposition. Therefore the wavefront decomposition segments set $\mathrm{X}$ into its branches (Serra, 1998; 2001).

Clearly, the mapping $x \rightarrow P(x)$ which associates with any point $x \in Z$ the tree diagram characterized by the proposition, depends on the choice of point $x$, even if, when considering the common meaning of a tree, the partition remains almost the same for all the points selected low enough in the trunk. Besides, in this case, the tree may be defined as a partition for which there is no confluence for a suitably selected origin $x$ (i.e. in the trunk).

Note that we are talking about connectivity here, and not about homotopy: in $\mathbb{R}^{3}$ particularly, the sections may show closed pores or toric holes.

\section{USE OF THE TREE DIAGRAM FOR EMBRYONIC KIDNEYS}

In order to illustrate the above matter, we propose to segment the first one of the two kidneys of Fig. 1. The analysis contains four steps:

1. set construction from the initial data;

2. geodesic distance function of a marker in the set;

3. extremities;

4. branches.

\section{BINARIZATION}

This simple operation only requires a thresholding between 60 and 255, followed with the fill-in of the bidimensional internal pores by geodesic dilation. Still, the main connected component has to be extracted. In order to do this, we take as marker $x$ one point at the beginning of the trunk. The reconstruction shows that the kidney tree diagram is broken into two disjoint parts at the level of section no. 14 (see Fig. 7b). This is caused by the inaccuracy of confocal microscopy. In order to put it right, both parts have been reconnected by a small closing that generates the flat zone visible on Fig. 7 a.
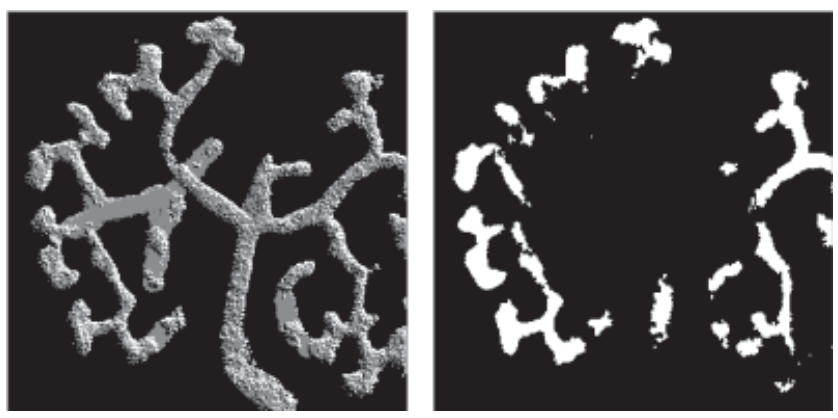

Fig. 7. a) Perspective view of the binarized kidney; $b$ ) confocal section no. 14.

\section{GEODESIC DISTANCE FUNCTION}

We take for marker $X$ the base of the tree, i.e. in the bottom-up sense, the content of the first section that is not empty. The geodesic distance function starts from marker $X$ at the base of the kidney and progresses inside the tree according to unit cube-octahedra (see Fig. 8)

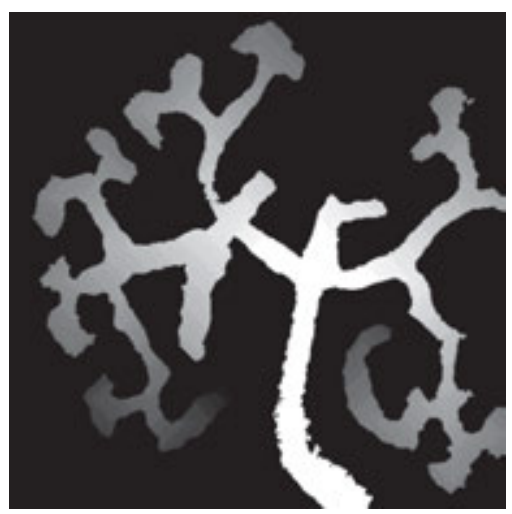

Fig. 8. Geodesic distance function from the anchorage set $X$ (negative view of the supremum of the sections).

\section{EXTREMITIES}

The extremities are nothing but the region maxima of the previous geodesic function. These ultimate eroded points are depicted on Fig. 9a, where number of quite unsignificant maxima can been observed. They correspond to some roughness of the 3-D surface rather than to actual extremities. Their areas are very 
small and they can be removed by a small surface opening (Fig. 9b). When using this algorithm in routine, we would better start with a regularization of the set under study by means of an isotropic tridimensional opening of size 1 or 2 , providing that it does not break the connectivity.
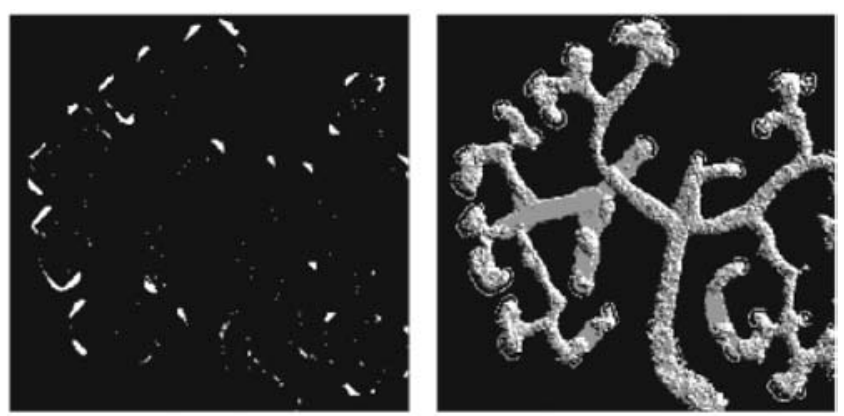

Fig. 9. a) All extremities of the kidney; b) Remaining extremities after a small area opening (surrounded zones), in superimposition with a perspective view of the kidney tree.

\section{BRANCHINGS}

The extraction of branchings, which is conceptually simple, may lead nevertheless to a consequent computing time. Considering the quite visible structure of the projected tree, the algorithm used below is slightly less precise, but faster and easier to implement.

In a first step, bidimensional branchings on the tree projection (i.e. on the 2D set of Fig. 10) are found, by following the progression of the $2 \mathrm{D}$ wavefront from the base of the trunk, and extracting each portion that has just been disconnected. The operation leads to Fig. 10. Then, we get back to the $3 \mathrm{D}$ space by building vertical cylinders whose bases are located at the 2D branchings (i.e. the white zones of Fig. 10), and slightly dilated (size 2). Finally, we take the intersection between these cylinders and the 3D tree.

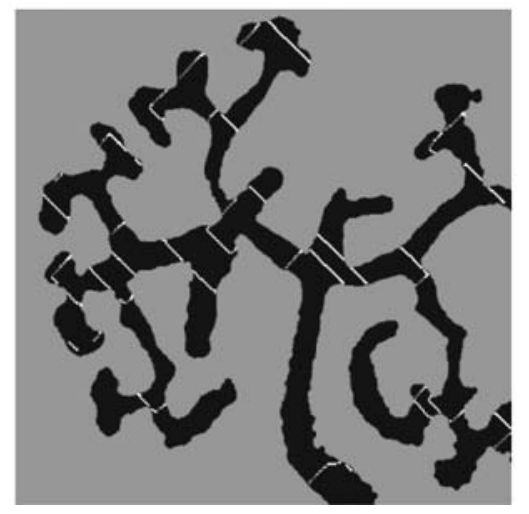

Fig. 10. Projection of the $3 D$ branchings.

\section{RESULTS}

Altogether, starting from the connected kidney tree, we got to its segmentation into disjoint branches separated by thin branchings. Some branches contain one or more, of the tree extremities. From such a segmentation, it becomes now possible to replace the object under study by a "tree" in the meaning of graph theory, where the edges can be weighted geometrical characteristics (volume, length, location of its center, possible end points ... etc).

\section{EULER-POINCARÉ NUMBER AND SPACE GRAPHS}

Historically, the Euler-Poincaré constant (in brief: EPC) appeared in two slightly different domains of mathematics. Firstly, there was Euler's reasoning about the relations between the polyhedrons vertices, edges and faces, which was formalized in terms of planar graphs by Cauchy. This way of thinking leads to counting algorithms, which are based on the elementary edges, squares and triangles (in the hexagonal grid). It extends to various cubic, cubeoctahedron and rhombo-dodecahedron of $\mathbb{R}^{3}$, without any particular theoretical difficulty, but with a growing heaviness of the elementary operations to be carried out.

The second way, Poincaré's, and Hadwiger's later on, links the successive definitions of EPC thanks to an induction holding on the dimensions of the space (Hadwiger, 1957; Bertrand, 1999). When transposed to a digital grid, this approach is limited to cubic (or to parallelepipedic) grids, but, in return, leads to a much simpler and faster expression than the graphs one. Thus, for a bounded digital set A, we have:

- In $\mathbb{Z}^{1}$,

$$
v_{1}(A)=N(\text { vertices })-N(\text { edges })=N(\bullet)-N(-)
$$

- $\quad$ In $\mathbb{Z}^{2}$, for the square grid:

$$
\begin{aligned}
v_{2}(A) & =N(\text { vertices })-N(\text { edges })+N(\text { faces }) \\
& =N(\bullet)-N(-)-N(\mid)+N(\sqsubset)
\end{aligned}
$$

Still in $\mathbb{Z}^{2}$, if we agree on calling $\overline{V_{1}}(A)$ the sum of the constants $v_{1}$ of the horizontal lines of $A$, we can see that

$$
v_{2}(A)=\overline{v_{1}}(A)-\overline{v_{1}}(A € \mid),
$$

where $A \ominus \mid$ stands for the Minkowski substraction of $A$ by the unit vertical segment. 


$$
\begin{aligned}
v_{3}(A)= & N(\bullet)-N(\square)-N(\not)+N(\square) \\
& -N(\mid)+N(\square)+N(\emptyset)-N(\square)
\end{aligned}
$$

Fig. 11. Euler-Poincaré Constant in $\mathbb{R}^{3}$.

In $\mathbb{Z}^{3}$, this is the same, and Euler's number $v_{3}(A)$ defined as

$$
\begin{aligned}
v_{3}(A)= & N(\text { vertices })-N(\text { edges })+ \\
& N(\text { faces })-N(\text { blocks })
\end{aligned}
$$

is expressed by the same increment as before, for we have (see Fig. 11)

$$
v_{3}(A)=\overline{v_{2}}(A)-\overline{v_{2}}(A \Theta \mid)
$$

where $\overline{v_{2}}(A)$ is the sum of Euler bidimensional numbers of the horizontal sections of $\mathrm{A}$, and where $\Theta$ stands for he Minkowski substraction of $A$ by the unit vertical segment (equation (2) can easily be extended to $\mathbb{R}^{n}$ by recurrence). Here the word "block" means "elementary cube of 8 voxels", as depicted in Fig. 11. Constant $v_{3}$ is independent of the choice of the "vertical" direction.

From an experimental point of view, the equation (2) is very convenient, for in image processing systems, Euler bidimensional constants are generally rapid to get and the unit linear erosion between two consecutive planes is a simple operation, too. It is this equation (2) that has been implemented in the shinbone example below.

Finally, remember that the EPC of a simply connected object (i.e. homeomorphic to a cube) equals 1 , that of a torus (typically, a donut) equals 0 , and that of a hollow sphere (such as a football) equals 2. More generally, in $3 \mathrm{D}$ the EPC is equal to the number of the connected blocks, minus the number of tunnels, plus the number of cavities. Moreover, the constant $v$ is $\mathrm{C}$ additive, which means that

$$
v(A)+v\left(A^{\prime}\right)=v\left(A\left\llcorner A^{\prime}\right)+v\left(A\left\ulcorner A^{\prime}\right),\right.\right.
$$

is an equation that allows one to reduce complex figures to the most simple ones. Thus, the EPC of lampshade pierced by 1000 pin holes equals -1000 .

\section{SHAFT OF CHICKEN EMBRYO SHINBONES}

\section{PURPOSE}

The bone zone under study is situated in the central part of a chicken embryo shinbone, whose axis defines the vertical. The experimental data form a series of 98 rectangular slices of $320 \times 310$ pixels each. The uniform grey of the shinbone phase allowing an easy thresholding (see Fig. 12a), the two problems to be solved are then the following:

1. Implementing and checking Dr Staub's model, that is to say switching from the model of nested cylinders to an effective segmentation of the bone into nested structures thanks to some convenient quantitative criterion (to be found);

2. Once the segmentation is achieved, extracting more specifically the bridges that link two successive cylinders, and calculating the homotopy of both bridges and cylinders.

\section{ALGORITHM}

For the sake of pedagogy, we work, on the one hand, on all 98 slices, and on the other hand, on the first 14 ones only. We call "bone" the first file, and "bone1" the second one. Thanks to reduced thickness file bone1, some structures are made more easily visible; moreover, the comparison between the wavefronts of bone and bone1 will inform us about the representativity of sample bone1.
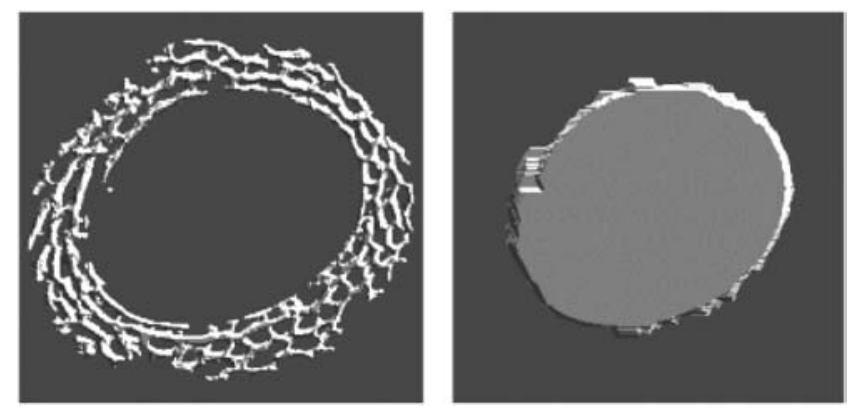

Fig. 12. a) Perspective view of bone1; b) Central cylindric marker $M$.

If the nested cylinders model is correct, the wavefront stemming from the central medulla zone and penetrating into the bone should propagate more rapidly when it floods a cylindrical crown than when it crosses the narrow isthmuses that link the crowns altogether. Therefore, we have to:

- generate a relevant central marker $M$;

- plot the curve of the wavefront surface $F(\lambda, M)$ versus distance $\lambda$, which should show oscillations with more or less periodic minima;

- decompose the geodesic wave into sections limited by minima values (bone segmentation); 
- extract the wavefront at each minimum, which will result into bridges;

- calculate Euler constant for bridge sets, and cylinders;

... all operations that will now be executed.

\section{RESULTS}

The central marker $\mathrm{M}$ is obtained by working one section after the other, and by extracting the central pore as a large connected particle that misses the edges (algorithm bone1), see Fig. 12b.

The measurement variation of the wavefront surface, for both files bone and bone1 is plotted in Fig. 13. Their minima are approximately on the same abcissae, for instance 16 instead of 13 or 6 instead of 5, which is an auspicious start. Bone and bone 1 segmentations, carried out from the following minima abcissae

$$
6 ; 22 ; 44 ;
$$
for bone

$5 ; 18,24 ; 41,65$; for bone 1

lead to the results shown in Fig. 14.

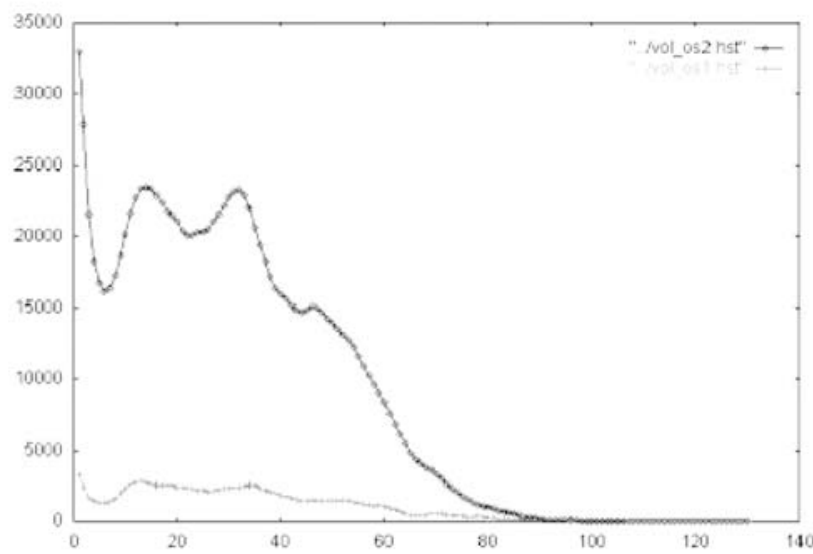

Fig. 13. Plot of the wavefront surface versus the propagation steps.
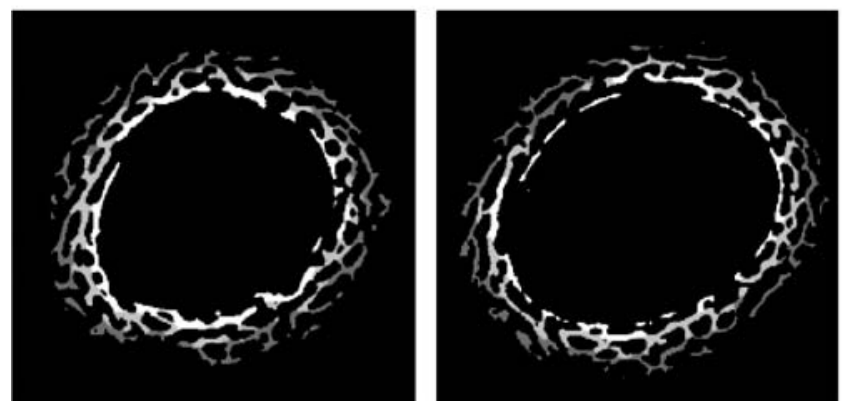

Fig. 14. 3D segmentations of two slices.
In order to extract the branchings between cylinders, a stronger and partially false hypothesis has to be made: the wavefronts corresponding to each minimum of the plot are supposed to be exclusively located in these narrows. Based on this approximation, the bridges between cylinders no. $m$ and $m+1$ match with the set difference between the $m+1$ and $m$ sizedgeodesic dilates, where $m$ is the abcissa of a minimum. The contact zones between the first two cylinders, for both files bone and bone1 are displayed in Fig. 15.
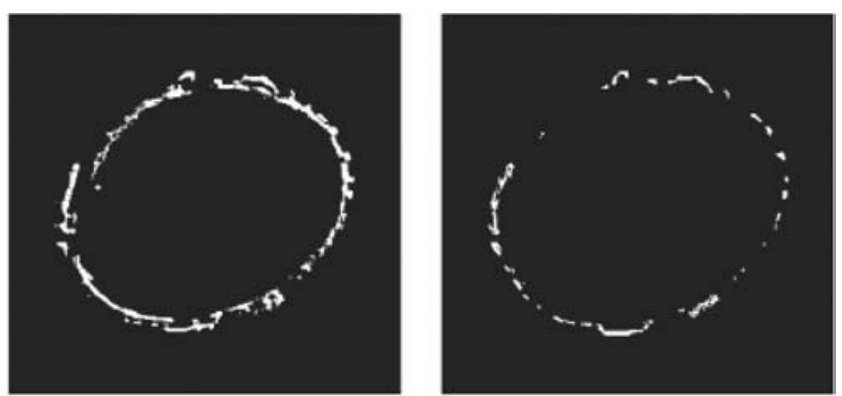

Fig. 15. Views from above of the dilated bridges, for "bone" and "bone 1".

We now treat the last point, about countings on the various extracted sets. As one can note on the following table, the bone is quite pierced and broken.

\begin{tabular}{lrr} 
Region & \multicolumn{2}{c}{$\begin{array}{c}\text { Euler Poincaré number } \\
\text { bone }\end{array}$} \\
& -1536 & -237 \\
bone1 & \\
initial bone & 1447 & -275 \\
$\begin{array}{l}\text { connected component, } \\
\text { adjacent to the marker }\end{array}$ & -1885 & 205 \\
$\begin{array}{l}\text { bridges between the first } \\
\text { two cylinders } \\
\text { same bridges, followed }\end{array}$ & 32 & 10
\end{tabular}
by a unit dilation

Broken, for when reducing the object to its part adjacent to the central marker, at least 1885 $1536=349$ small isolated parts are removed (experimental artifacts?). Pierced, for this main connected component has 1885 holes, if it is admitted that it does not contain internal closed pores (this seems realistic when we consider the thicknesses).

The EPC of the bridges (1447) seems quite high. In fact, more than a single thick wire linking one cylinder to the following one, a joint rather looks like a bundle of fine fibers. This is the reason why an elementary dilation (the 13 voxels of the unit cubeoctahedron) brings Euler-Poincaré number down from 1447 to 32 . 


\section{CONCLUSION}

Remarkably, the same wavefront concept in $\mathbb{R}^{n}$ or $\mathbb{Z}^{n}$ allows one to describe:

- the connected components, via its surface measurement;

- the bottlenecks, via the minima of its variation;

- the branches, via the variation of its connectivity;

- and the extremities, via its ultimate locations; and its application to complex 3-D histologic structures proves the outstanding power of this tool.

\section{ACKNOWLEDGEMENT}

I thank on the one hand, Dr J. Bertram, and on the other hand Dr Staub and M. M. Mendjeli, for the discussions we had together, and for the digital data they kindly gave me. I also thank M. Ch. Bernard for his comments about sequential monotone convergence of connected compact sets. I did not use them here, but this talk helped me in the relaunching of this writing. I thank also M. G. Fricout: thanks to his different point of view about the study of kidney tress, he forced me to explain my own position more clearly. Finally, I thank the reviewer of this paper for his help.

\section{REFERENCES}

Clark AT, Young RJ, Bertram JF (2001). In vitro studies on the roles of transforming growth factor-beta1 in rat metaphrenic development, Kidney Int. 59:1641-53.
Bertrand G (1999). New Notions for Discrete Topology. In: Bertrand G, Couperie M, Perroton L, eds. Discrete Geometry for Computer Imagery, Lecture notes in computer sciences, Vol 1568. Paris: Springer.

Choquet G (1966). Topologie. Paris: Masson.

Fricout G, Cullen-Mc Ewen LA, Harper IA, Jeulin D, Bertram JF (2002). A quantitative method for analysing 3D branching in embryonic kidneys: development of a technique and preliminary data. Image Anal Sterol 21:37-41.

Hadwiger H (1957). Vorselungen über Inhalt, Oberfläche und Isoperimetrie. Berlin: Springer.

Klein JC (1976). Conception et réalisation d'une unité logique pour l'analyse quantitative d'images. Thesis, University of Nancy.

Lantuejoul C, Beucher S (1981). On the use of the geodesic metric in image analysis. J Microsc 121:39-49.

Meyer F (1976). Tech. report CMM, Ecole des Mines de Paris.

Meyer F (1992). Mathematical Morphology: from two dimensions to three dimensions. J Microsc 165:5-29.

Serra J, ed. (1988). Image Analysis and Mathematical Morphology. Vol. 2: Theoretical Advances. London: Academic Press.

Serra J (1998). Connectivity on complete lattices. J Math Imaging Vision 9:231-51.

Serra J (1997). Cube, cube-octahedron or rhombododecahedron as bases for 3-D shape descriptions. In: Arcelli C, Sanniti di Baja G, Cordella LP, eds. Advances in Visual Form Analysis, Singapore: World Scientific, 502-19.

Serra J (2001). Connexions et segmentation d'image. Int. Report Ecole des Mines, N-16/02/MM. 